\title{
Feasibility of Convolutional Neural Networks (CNN) for Content Based Image Retrieval of Images from Hadoop
}

\author{
Muthiah.M.A ${ }^{\mathrm{a}, 1}$, Logashanmugam.E ${ }^{\mathrm{b}}$, and Nandhitha.N.M ${ }^{\mathrm{c}}$ \\ ${ }^{a}$ Research scholar, School of Electrical and Electronics, Sathyabama Institute of \\ Science and Technology, Chennai \\ ${ }^{\mathrm{b}}$ Professor, School of Electrical and Electronics, Sathyabama Institute of Science and \\ Technology, Chennai \\ ${ }^{\mathrm{c}}$ Professor, School of Electrical and Electronics, Sathyabama Institute of Science and \\ Technology, Chennai
}

\begin{abstract}
In search engines, though feature-based query is provided, Content Based Image Retrieval (CBIR) still results in less sensitivity and specificity. It is because the conventional approach is based on feature extraction and inherent parameters in conventional feed forward networks. Performance of the system is strongly dependent on the extracted features. Hence it is necessary to develop a CBIR system that retrieves the similar images without explicit feature extraction. Convolutional Neural Networks are the recent neural network architectures which accept images as input and perform both feature extraction and classification. The proposed work aims at using the conventional architectures of VGGNET, RESNET, and DENSENET for flower classification in CBIR. Performance is measured in terms of accuracy of classification.
\end{abstract}

Keywords. CBIR, Deep learning, Accuracy, Big data, Hadoop.

\section{Introduction}

Image classification for CBIR is an important application of digital image processing. Initially the image database is collected and the images are studied. Having understood the metadata, features must be identified for representing the images. Real challenge lies in identifying the correct features for that particular application. Various features namely shape features, statistical features, texture features are cited in the literature. These features or a new set of image specific features must be identified. Having identified the features, the next task is to check the suitability of these features. These features must be similar for the same set of images and must be different between sets i.e less intra class variance and high interclass variance. This criterion is difficult to achieve in case of real time images and hence research continues in this area of feature engineering.

\footnotetext{
${ }^{1}$ Muthiah.A, Research Scholar, School of Electrical and Electronics Sathyabama Institute of Science and Technology, Chennai, India.

Email:Imuthiah@gmail.com ${ }^{1}$
} 
Once satisfied with the features, these features are then given as inputs to either unsupervised or supervised algorithms for classification. Though the accuracy of classification is dependent on the classification algorithm, its inherent parameters, it is strongly dependent on the extracted features. So the paradigm has shifted to algorithms which perform feature extraction and classification. Such networks are called Deep learning networks as the depth of the network is in the order of $50 \mathrm{~s}$ and $100 \mathrm{~s}$, i.e. the number of layers in these networks is more. Deep learning Networks are available for both signal analysis (Recurrent Neural Network, Long Short Term Memory recurrent network) and image analysis (Convolutional Neural Networks). Convolutional Neural Networks are called so because they use convolution operator to extract the features. First few layers of the network extract the discontinuities and the further layers combine these discontinuities to form the features describing the input images. Also in contrast to fully connected layers in Back Propagation Network (BPN), the connection is sparse i.e not all the neurons in the previous layers are connected to all the neurons in the next layers. This sparseness protects the network from overloading. Once the features are extracted, positive values are retained as such and all the negative values are converted to zeroes using Rectified Linear Unit (ReLU). Reduced feature map is then obtained using pooling layers.

\section{Related Work}

In $\mathrm{CNN}$, based on the filters used for convolution, the number of layers, fully connected networks, multiple CNN architectures like ALEXNET, GOGLENET, VGGNET, RESNET, DENSENET, SQUEEZENET sprang into existence. ThiThanhNhan Nguyen et al (2016) found that Googlenet is the best suited architecture fr flower database classification with an accuracy of $(90.82 \%)$. Y. Liu et al (2016) achieved an accuracy of $84.2 \%$ with their own CNN architecture that had three fully connected networks. Abu et al (2019) achieved an accuracy of 90\% classification for flower database classification with their own CNN model. However, only five sets of flowers were considered for classification. I. Gogul and V. S. Kumar (2017) proved that $\mathrm{CNN}$ outperforms conventional feature extraction and classification algorithms for flower database. Arwatchananukul (2020) used CNN for the classification of 15 types of orchids. The sample size was 100 for each type amounting to a total of 1500 images. Accuracy of classification is greater than $95 \%$. It is thus necessary to come out with a $\mathrm{CNN}$ architecture that outperforms the networks cited in the literature for a very large dataset.

\section{Research Database}

In this work, flower dataset is taken which consists of 102 (one hundred and two) different categories of flowers used for processing and classification based on extracted features. Each category is named as A1, A2 ... and so on till A102. Each category consists of certain number of images with a minimum of 20 each in every category. On a total, there are 8189 flower images in 102 categories. The same dataset is used for all the different types of Convolutional Neural Networks (CNN). 50\% of the dataset is used for training and $50 \%$ is used for testing. Dataset is stored in hadoop file system 
where hadoop is used for storing the database from where it is used for training and testing the neural networks.

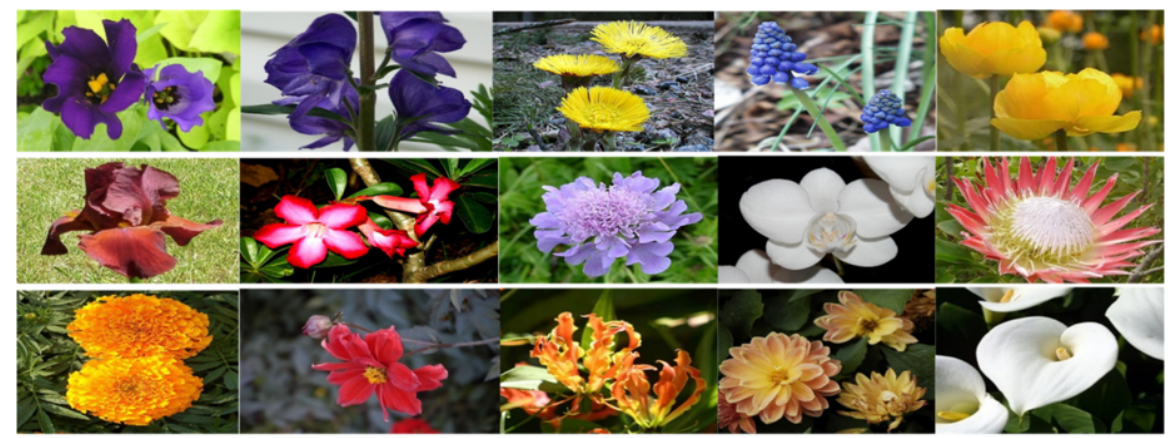

Figure 1.Images from flower database (Courtesy: https:// www.robots.ox.ac.uk/ vgg/ data/ flowers)

\section{CNNFor CBIRof Images From Hadoop}

In this work, a hadoop cluster with 8 systems is created with one as the master node and the remaining 7 (seven) as slave nodes. In master there is no data node to store the data only slave nodes will have data node to store data. Every operation has to be done only in master system. Master node consists of metastore which have all information about data where it has stored. Figure 2 shows the details of cluster.

\section{Datanode usage histogram}

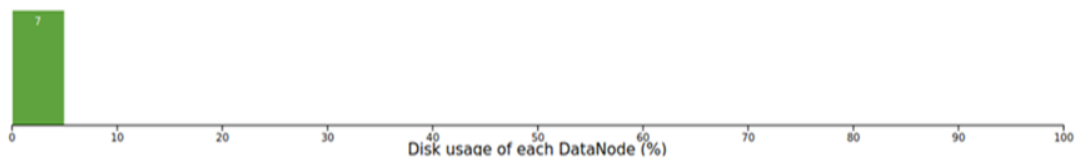

In operation

\begin{tabular}{|c|c|c|c|c|c|c|c|c|c|c|}
\hline Node & Last contact & Admin State & Capacity & Used & Non DFS Used & Remaining & Blocks & Block pool used & Falled Volumes & Versio \\
\hline slave 3:50010 (10.0.3.107:50010) & 0 & In Service & $78.24 \mathrm{~GB}$ & $380.4 \mathrm{MB}$ & $53.35 \mathrm{~GB}$ & $20.51 \mathrm{~GB}$ & 8236 & 380.4 MB $(0.47 \%)$ & 0 & 2.7 .7 \\
\hline slave6:50010 (10.0.3.85:50010) & 1 & In Service & $78.24 \mathrm{~GB}$ & 381.24 MB & $53.46 \mathrm{~GB}$ & $20.39 \mathrm{~GB}$ & 8214 & $381.24 \mathrm{MB}(0.48 \%)$ & 0 & 2.7 .7 \\
\hline slave7:50010 (10.0.3.88:50010) & 1 & in Service & $78.24 \mathrm{~GB}$ & $380.84 \mathrm{MB}$ & $53.26 \mathrm{~GB}$ & $20.59 \mathrm{~GB}$ & 8235 & $380.84 \mathrm{MB}(0.48 \%)$ & 0 & 2.7 .7 \\
\hline stave2: 50010 (10.0.3.108:50010) & 1 & In Service & $78.24 \mathrm{~GB}$ & $381.12 \mathrm{MB}$ & $53.51 \mathrm{~GB}$ & $20.34 \mathrm{~GB}$ & 8214 & $381.12 \mathrm{MB}(0.48 \%)$ & 0 & 2.7 .7 \\
\hline slave4:50010 (10.0.3.91:50010) & 1 & in Service & $78.24 \mathrm{~GB}$ & $380.49 \mathrm{MB}$ & $53.38 \mathrm{~GB}$ & $20.47 \mathrm{~GB}$ & 8207 & $380.49 \mathrm{MB}(0.47 \%)$ & 0 & 2.7 .7 \\
\hline slaves:50010 (10.0.3.82:50010) & 1 & In Service & $78.24 \mathrm{~GB}$ & $380.97 \mathrm{MB}$ & $53.34 \mathrm{~GB}$ & $20.52 \mathrm{~GB}$ & 8209 & $380.97 \mathrm{MB}(0.48 \%)$ & 0 & 2.7 .7 \\
\hline slave1:50010 (10.0.3.109:50010) & 0 & In Service & $78.24 \mathrm{~GB}$ & $380.54 \mathrm{MB}$ & $54.08 \mathrm{~GB}$ & $19.77 \mathrm{~GB}$ & 8208 & $380.54 \mathrm{MB}(0.47 \%)$ & 0 & 2.7 .7 \\
\hline
\end{tabular}

Figure 2.Details of the cluster

\subsection{Convolutional Neural Networks for flower database classification}

In this work, VGGNET, RESNET and DENSENET are used for the classification of flower images. All these networks use convolutional layers followed by activation layer (Rectified Linear Unit) and pooling layers. At the end, a fully connected network 
is used for classifying the flowers into groups. An overview of these networks is given in Table 1. Irrespective of the size of the original images, all the images are augmented as $224 \times 224 \times 3$. It means that all the input images are resized with 224 rows, 224 columns and are three dimensional (colour images). It simplifies the convolution operation when a common filter is applied across each and every element.

Table 1.VGGNET, RESNET and DENSENET - An Overview

\begin{tabular}{|c|c|c|c|c|c|c|}
\hline $\begin{array}{l}\text { Name of the } \\
\text { network }\end{array}$ & VGG16 & VGG19 & $\begin{array}{l}\text { RESNET } \\
101\end{array}$ & $\begin{array}{l}\text { RESNET } \\
50\end{array}$ & $\begin{array}{l}\text { RESNET } \\
18\end{array}$ & Densenet201 \\
\hline $\begin{array}{l}\text { Number of } \\
\text { layers }\end{array}$ & $\begin{array}{l}16 \text { layers } \\
\text { deep }\end{array}$ & $\begin{array}{l}\text { 19layers } \\
\text { deep }\end{array}$ & $\begin{array}{l}101 \text { layers } \\
\text { deep and can } \\
\text { classify } \\
\text { images into } \\
1000 \text { object } \\
\text { categories }\end{array}$ & $\begin{array}{l}50 \text { layers } \\
\text { deep and can } \\
\text { classify } \\
\text { images into } \\
1000 \text { object } \\
\text { categories }\end{array}$ & $\begin{array}{l}18 \text { layers } \\
\text { deep and can } \\
\text { classify } \\
\text { images into } \\
1000 \text { object } \\
\text { categories }\end{array}$ & $\begin{array}{l}201 \text { layers } \\
\text { deep and can } \\
\text { classify } \\
\text { images into } \\
1000 \text { object } \\
\text { categories }\end{array}$ \\
\hline $\begin{array}{l}\text { Input to the } \\
\text { subsequent } \\
\text { convolutional } \\
\text { layers }\end{array}$ & $\begin{array}{l}\text { Input is } \\
\text { received } \\
\text { only from } \\
\text { the } \\
\text { immediately } \\
\text { preceding } \\
\text { layer (no } \\
\text { short cuts) }\end{array}$ & $\begin{array}{l}\text { Input is } \\
\text { received } \\
\text { only from } \\
\text { the } \\
\text { immediately } \\
\text { preceding } \\
\text { layer (no } \\
\text { short cuts) }\end{array}$ & $\begin{array}{l}\text { Input is } \\
\text { received } \\
\text { only from } \\
\text { the } \\
\text { immediately } \\
\text { preceding } \\
\text { layer (with } \\
\text { short cuts) }\end{array}$ & $\begin{array}{l}\text { Input is } \\
\text { received } \\
\text { only from } \\
\text { the } \\
\text { immediately } \\
\text { preceding } \\
\text { layer (with } \\
\text { short cuts) }\end{array}$ & $\begin{array}{l}\text { Input is } \\
\text { received } \\
\text { only from } \\
\text { the } \\
\text { immediately } \\
\text { preceding } \\
\text { layer (with } \\
\text { short cuts) }\end{array}$ & $\begin{array}{l}\text { Input is } \\
\text { received from } \\
\text { all the } \\
\text { preceding } \\
\text { layers }\end{array}$ \\
\hline $\begin{array}{l}\text { Transition } \\
\text { layer } \\
\text { operation }\end{array}$ & $\begin{array}{l}\text { Sum of } \\
\text { residues } \\
\text { (element } \\
\text { wise } \\
\text { addition) }\end{array}$ & $\begin{array}{l}\text { Sum of } \\
\text { residues } \\
\text { (element } \\
\text { wise } \\
\text { addition) }\end{array}$ & $\begin{array}{l}\text { Sum of } \\
\text { residues } \\
\text { (element } \\
\text { wise } \\
\text { addition) }\end{array}$ & $\begin{array}{l}\text { Sum of } \\
\text { residues } \\
\text { (element } \\
\text { wise } \\
\text { addition) }\end{array}$ & $\begin{array}{l}\text { Sum of } \\
\text { residues } \\
\text { (element } \\
\text { wise } \\
\text { addition) }\end{array}$ & $\begin{array}{l}\text { Concatenation } \\
\text { of feature } \\
\text { maps (feature } \\
\text { map } \\
\text { operation) }\end{array}$ \\
\hline $\begin{array}{l}\text { Pooling } \\
\text { layers }\end{array}$ & $\begin{array}{l}\text { Every } \\
\text { convolution } \\
\text { layer has a } \\
\text { pooling } \\
\text { layer }\end{array}$ & $\begin{array}{l}\text { Every } \\
\text { convolution } \\
\text { layer has a } \\
\text { pooling } \\
\text { layer }\end{array}$ & $\begin{array}{l}\text { Only two } \\
\text { pooling } \\
\text { layers (one } \\
\text { at the } \\
\text { beginning } \\
\text { and the other } \\
\text { at the end) }\end{array}$ & $\begin{array}{l}\text { Only two } \\
\text { pooling } \\
\text { layers (one } \\
\text { at the } \\
\text { beginning } \\
\text { and the other } \\
\text { at the end) }\end{array}$ & $\begin{array}{l}\text { Only two } \\
\text { pooling } \\
\text { layers (one } \\
\text { at the } \\
\text { beginning } \\
\text { and the other } \\
\text { at the end) }\end{array}$ & $\begin{array}{l}\text { Every } \\
\text { convolution } \\
\text { layer has a } \\
\text { pooling layer }\end{array}$ \\
\hline Speed & Slow & slow & Fast & fast & fast & fastest \\
\hline
\end{tabular}

Performance of the above said networks on the classification of flower database is shown in Table 2 for test images of fl category. There are mismatches between the desired and the actual outputs of the Convolutional Neural Networks used in this work. Overall performance of the networks for the classification of test images is measured in terms of accuracy and is given in Table 2 
Table 2. VG Relationship between actual and predicted classes for fl from various $\mathrm{CNN}$ architectures

\begin{tabular}{|c|c|c|c|c|c|c|}
\hline Desired & $\begin{array}{l}\text { Column2 } \\
\text { VGG16 } \\
\end{array}$ & $\begin{array}{l}\text { Column3 } \\
\text { VGG19 } \\
\end{array}$ & RESNET18 & RESNET50 & RESNET101 & Densenet201 \\
\hline $\mathrm{fl}$ & f45 & f64 & $\mathrm{fl}$ & f55 & $\mathrm{fl}$ & f1 \\
\hline $\mathrm{fl}$ & $\mathrm{fl}$ & $\mathrm{fl}$ & $\mathrm{f} 51$ & $\mathrm{fl}$ & $\mathrm{f} 51$ & $\mathrm{fl}$ \\
\hline $\mathrm{fl}$ & $\mathrm{fl}$ & $\mathrm{fl}$ & f93 & $\mathrm{fl}$ & $\mathrm{f} 93$ & $\mathrm{fl}$ \\
\hline $\mathrm{fl}$ & $\mathrm{fl}$ & $\mathrm{fl}$ & $\mathrm{fl}$ & $\mathrm{fl}$ & $\mathrm{fl}$ & $\mathrm{fl}$ \\
\hline $\mathrm{fl}$ & $\mathrm{fl}$ & $\mathrm{fl}$ & f86 & $\mathrm{fl}$ & f86 & $\mathrm{f} 1$ \\
\hline $\mathrm{fl}$ & $\mathrm{fl}$ & $\mathrm{fl}$ & f51 & $\mathrm{fl}$ & $\mathrm{f} 51$ & $\mathrm{fl}$ \\
\hline $\mathrm{fl}$ & f97 & f51 & $\mathrm{f} 1$ & $\mathrm{fl}$ & $\mathrm{fl}$ & $\mathrm{f} 1$ \\
\hline $\mathrm{fl}$ & f34 & $\mathrm{fl}$ & $\mathrm{fl}$ & $\mathrm{fl}$ & $\mathrm{fl}$ & $\mathrm{f} 95$ \\
\hline $\mathrm{fl}$ & f53 & f96 & $\mathrm{f} 40$ & $\mathrm{fl}$ & $\mathrm{f} 40$ & $\mathrm{fl}$ \\
\hline $\mathrm{fl}$ & f1 & $\mathrm{fl}$ & f86 & $\mathrm{fl}$ & f86 & $\mathrm{f} 53$ \\
\hline $\mathrm{fl}$ & f96 & $\mathrm{fl}$ & f51 & f34 & $\mathrm{f} 51$ & $\mathrm{fl}$ \\
\hline $\mathrm{fl}$ & f53 & f51 & f51 & $\mathrm{fl}$ & $\mathrm{f} 51$ & f98 \\
\hline $\mathrm{fl}$ & f96 & f51 & $\mathrm{f} 40$ & $\mathrm{fl}$ & $\mathrm{f} 40$ & $\mathrm{fl}$ \\
\hline $\mathrm{fl}$ & f97 & f51 & fl & f1 & $\mathrm{fl}$ & $\mathrm{f} 1$ \\
\hline $\mathrm{fl}$ & f98 & f86 & fl & f51 & $\mathrm{fl}$ & f98 \\
\hline $\mathrm{fl}$ & $\mathrm{f} 1$ & f86 & $\mathrm{fl}$ & $\mathrm{fl}$ & $\mathrm{f} 1$ & $\mathrm{f} 98$ \\
\hline $\mathrm{fl}$ & f34 & f86 & $\mathrm{f} 1$ & f51 & $\mathrm{fl}$ & $\mathrm{fl}$ \\
\hline $\mathrm{fl}$ & f67 & f51 & $\mathrm{f} 1$ & f51 & fl & $\mathrm{fl}$ \\
\hline $\mathrm{fl}$ & $\mathrm{fl}$ & f86 & f51 & f51 & f51 & $\mathrm{f} 1$ \\
\hline
\end{tabular}

Table 3. Performance of Convolutional Neural Networks for the classification of flowers in flowers database

\begin{tabular}{ccccccc}
\hline Parameters & VGG16 & VGG19 & RESNET18 & RESNET50 & RESNET101 & Densenet201 \\
$\begin{array}{c}\text { No. of matches } \\
\text { No. of }\end{array}$ & 3362 & 2694 & 3443 & 3656 & 3633 & 3770 \\
$\begin{array}{c}\text { mismatches } \\
\begin{array}{c}\text { Total no. of } \\
\text { images }\end{array}\end{array}$ & 4070 & 4070 & 4070 & 4070 & 4070 & 4070 \\
$\begin{array}{c}\text { Accuracy in } \\
\text { percentage }\end{array}$ & $82.6 \%$ & $66.19 \%$ & $84.59 \%$ & $89.8 \%$ & $89.2 \%$ & $92.6 \%$ \\
\hline
\end{tabular}

From the last row of Table 3, it is evident that DENSENET outperforms other networks in classifying the flower images with an accuracy of $92.6 \%$ as it considers concatenation of feature mapping instead of summation.

\section{Conclusion and Future Work}

In this work, feasibility of Convolutional Neural Networks for the classification of flower images is studied. Of the various Convolutional Neural Network architectures, 
VGG16 has an accuracy of $82.60 \%$ respectively. VGG19 has accuracy of $66.19 \%$ respectively. DENSENET201 has an accuracy of $92.6 \%$ respectively. RESNET18 has an accuracy of $84.59 \%$ respectively. RESNET50 has an accuracy of $89.8 \%$ respectively. RESNET101 has an accuracy of 89.2\% respectively. For the classification of flower dataset, Densenet201 has the highest accuracy. Though the proposed algorithms are scalable in nature, the impact of large dataset on the performance of the proposed techniques can be studied. The work has stopped at simulation level. Also new architectures and image specific improvements can be made in the existing techniques to improve the performance of the network.

\section{References}

[1] Nguyen, Nhan\& Le, Van \& Le, Thi\&Hai, Vu \&Pantuwong, Natapon\&Yagi, Yasushi. (2016) Flower species identification using deep convolutional neural networks. Proc. of Regional Conference on Computer and Information Engineering (RCCIE 2016)

[2] Liu.Y, Tang.F, Zhou.D , Y. Meng and W. Dong. Flower classification via convolutional neural network. 2016 IEEE International Conference on Functional-Structural Plant Growth Modeling, Simulation, Visualization and Applications (FSPMA), Qingdao, 2016, pp. 110-116, doi: 10.1109/FSPMA.2016.7818296.

[3] Abu, MohdAzlan\&Indra, NurulHazirah \& AbdRahman, Abdul \&Sapiee, Nor\& Ahmad, Izanoordina. (2019). A study on Image Classification based on Deep Learning and Tensorflow. 12. 563-569.

[4] Gogul and V. S. Kumar.Flower species recognition system using convolution neural networks and transfer learning. 2017 Fourth International Conference on Signal Processing, Communication and Networking (ICSCN), Chennai, 2017, pp. 1-6, doi: 10.1109/ICSCN.2017.8085675.

[5] Arwatchananukul, Sujitra\&Kirimasthong, Khwunta\&Aunsri, Nattapol. (2020). A New Paphiopedilum Orchid Database and Its Recognition Using Convolutional Neural Network. Wireless Personal Communications. 115. 10.1007/s11277-020-07463-3..

[6] Ambeth Kumar.V.D, S. Sharmila, Abhishek Kumar, A. K. Bashir, Mamoon Rashid, Sachin Kumar Gupta \&Waleed S. Alnumay .A novel solution for finding postpartum haemorrhage using fuzzy neural techniques. Neural Computing and Applications (2021) (https://doi.org/10.1007/s00521-02005683-z)

[7] AnkitKumar,VijayakumarVaradarajan,AbhishekKumar, PankajDadheech, SurendraSinghChoudhary, V.D. AKumar, B.K.Panigrahi, KalyanaC.Veluvolug, " Black hole attack detection in vehicular adhoc network using secure AODV routing algorithm”,Microprocessors and Microsystems, In Press,(https://doi.org/10.1016/j.micpro.2020.103352)

[8] Ambeth Kumar S. Malathi R. Venkatesan K Ramalakshmi, Weiping Ding, Abhishek Kumar . Exploration of an innovative geometric parameter based on performance enhancement for foot print recognition. Journal of Intelligent and Fuzzy System , vol. 38, no. 2, pp. 2181-2196, 2020.

[9] V.D.AK et al, (2016) .Human Life Protection In Trenches Using Gas Detection System. for the Journal of Biomedical Research. Vol.27 (2): 475-484

[10] Malathi .S et.al .Performance Improvement Using an Automation System for Segmentation of Multiple Parametric Features Based on Human Footprint.”,Journal of Electrical Engineering \& Technology , vol.10, no.4, pp.1815-1821, 2015. [http://dx.doi.org/10.5370/JEET.2015.10.4.1815]

[11] V.D.Ambeth Kumar et,al, "Enhancement in Footprint Image using Diverse Filtering Technique" Procedia Engineering journal, Volume 8, No.12, 1072-1080, 2012. [doi:10.1016/j.proeng.2012.01.965]

[12] S.V. Ruphitha et.al, " Management of Major Postpartum Haemorrhage by using Zigbee protocol - A Review “, 2021 6th International Conference on Inventive Computation Technologies (ICICT) (DOI: 10.1109/ICICT50816.2021.9358757)

[13] M. Indhumathi et.al , "Healthcare Management of Major Cardiovascular Disease-A review", 2021 6th International Conference on Inventive Computation Technologies (ICICT), (DOI: 10.1109/ICICT50816.2021.9358519)

[14] K. Sabarinathan et.al ., " Machine Maintenance Using Augmented Reality”, 3rd International Conference on Communication and Electronics Systems (ICCES), 2018. (DOI: 10.1109/CESYS.2018.8723900) 